\title{
Eight months of continuous positive airway pressure (CPAP) decrease tumor necrosis factor alpha (TNFA) in men with obstructive sleep apnea syndrome
}

\author{
Andrea Hegglin • Otto D. Schoch • Wolfgang Korte • \\ Kirsten Hahn • Christoph Hürny • Thomas Münzer
}

Received: 20 September 2010 /Revised: 10 March 2011 /Accepted: 11 March 2011 /Published online: 30 March 2011

(C) Springer-Verlag 2011

\begin{abstract}
Objectives The aim of this study was to assess serum tumor necrosis factor alpha (TNFA) concentrations 8 months of continuous positive airway pressure (CPAP) therapy.

Design This study used prospective, observational clinical trial. Patients Sixty-six patients with newly diagnosed sleep apnea syndrome (12 women, 54 men), age $52.3 \pm 9.8$ $($ mean \pm SD) with a body mass index of $29.7 \pm 4.4$ and an apnea-hypopnea index of $39.7 \pm 26.8$, were studied.

Intervention CPAP was administered for a mean of $7.8 \pm$ 1.3 months.
\end{abstract}

This study was funded by grants from the Institute for Clinical Chemistry and Hematology of the Kanton of St. Gallen to WK and $\mathrm{KH}$ and the Swiss National Science Foundation (SNF) Grant Number $3200-068115$ to TM, ODS and $\mathrm{CH}$

A. Hegglin · C. Hürny $\cdot$ T. Münzer

Geriatrische Klinik, Kompetenzzentrum Gesundheit und Alter,

St. Gallen, Switzerland

W. Korte $\cdot$ K. Hahn

Institute for Clinical Chemistry Kantonsspital St. Gallen,

St. Gallen, Switzerland

O. D. Schoch

Multidisciplinary Center for Sleep Medicine,

Kantonsspital St. Gallen,

St. Gallen, Switzerland

W. Korte $\cdot$ T. Münzer

University of Bern,

Bern, Switzerland

T. Münzer $(\bowtie)$

Geriatrische Klinik,

Rorschacherstrasse 94,

9000 St. Gallen, Switzerland

e-mail: thomas.muenzer@geriatrie-sg.ch
Measurements and results TNFA concentrations using an ultrasensitive ELISA assay at baseline and follow-up. TNFA decreased in men with high $(5.2 \pm 1.7 \mathrm{~h} /$ night, $-0.46 \pm 1.1 \mathrm{ng} / \mathrm{l}, p=0.001)$ and with low $(2.5 \pm 1.0 \mathrm{~h} / \mathrm{night}$ $-0.63 \pm 0.77 \mathrm{ng} / 1, p=0.001)$ adherence but not in women. Average number of hours of CPAP use correlated positively with delta TNFA $\left(R^{2} 0.08, p=0.04\right)$

Conclusion Long-term CPAP positively affects TNFA even in men with poor adherence to CPAP.

Keywords Obstructive sleep apnea syndrome - CPAP. Tumor necrosis factor $\cdot$ Inflammation $\cdot$ Adherence

\section{Introduction}

Proinflammatory cytokines such as tumor necrosis factor alpha (TNFA) play an important role as physiologic regulators of human sleep [1]. Obstructive sleep apnea syndrome (OSAS) is characterized by frequent disruptions of sleep [2] and leads to an alteration of the circadian rhythm of TNFA concentrations with a shift from nocturnal peaks to daytime peaks thereby aggravating daytime sleepiness [3]. Several reports have suggested that OSAS is associated with an increased proinflammatory activity indicated by elevations of C-reactive protein (CRP) [4], TNFA, and interleukin 6 (IL-6) $[5,6]$. Current research suggests that one factor contributing to increased inflammatory activity is visceral adipose tissue [7].

Three weeks of administration of a TNFA antagonist significantly decreased TNFA serum concentration and daytime sleepiness in OSAS patients [8] and therapy of OSAS with nasal continuous positive airway pressure (CPAP) decreased serum TNFA concentrations after 4 [9], 
8 [10], and 12 weeks [11]. Furthermore, short-term CPAP use significantly reduced visceral fat mass [12], thus potentially affecting TNFA-producing tissue. In contrast, we and others $[13,14]$ were not able to reproduce such an effect using a different imaging method. Thus, the contribution of visceral fat mass to TNFA concentrations in patients with OSAS remains unclear.

While a recent study reported a significant decline in CRP concentration [15] with a steep decline after three and a plateau after 6 months, little is known on the effects of longer-term CPAP therapy on TNFA. In addition, the contribution of other clinically relevant parameters such as disease severity and compliance with CPAP on changes in TNF concentrations in OSAS patients has not been examined.

In the current study, we aimed to examine if CPAP use of more than 6 months would decrease TNFA levels in women and men with newly diagnosed sleep apnea syndrome independent of relevant changes in adipose tissue.

\section{Methods}

\section{Inclusion and exclusion criteria}

A detailed description of our study population has been published previously [13]. In brief, patients with diabetes and hypercholesterolemia were included if on stable (oral) medication. None of the study participants had active inflammatory disease. There was no change in medication throughout the study period. Patients with pituitary disease, untreated thyroid disease, medication known to interfere with TNFA levels, and patients with elective surgery (such as gastric banding or hip replacement) within 6 months after enrolment were also not eligible. All patients were asked to keep their diets and their level of physical activity constant during the study period. This recommendation was given at baseline and reinforced at a follow-up visits after 1 and 3 months of CPAP. Written informed consent was obtained from all participants. The protocol had been approved by the Kanton St. Gallen Ethics Committee.

Patients were scheduled for an outpatient visit with one of the physicians in the sleep center. Prior to this appointment, they routinely completed a set of questionnaires. Covered domains were socio-demographic data, assessment of smoking status and alcohol use, a validated German version of the Epworth Sleepiness Scale (ESS) [16], and the Stanford Sleep Disorders Questionnaire [17]. During the appointment, each patient had a complete medical history to assess comorbidities [18] such as hypertension, coronary heart disease, diabetes, hypercholesterolemia, active inflammatory disease, and current medications. Based on the original paper, information on comorbidities were scored, and we calculated a summary score for each patient. Physical examination included inspection of the oropharynx and auscultation of the lungs and heart. Patients who, based on clinical judgment and the results of the screening questionnaires had a high likelihood to have OSAS, were then scheduled for polysomnography (PSG). In the evening, preceding PSG patients were asked to participate in the study. After an overnight fast, blood samples for baseline measurements (serum electrolytes, glucose, creatinine) including TNFA were drawn, independent of the result of the sleep study. Patients with OSAS who agreed to start on CPAP were scheduled for baseline body composition within 2 weeks after initiation of CPAP therapy. They received an instruction in nasal CPAP use for three consecutive nights in the hospital (including CPAP pressure titration with an automated device and pulse oximetry) followed by home CPAP. Monitoring of hours of operation of the CPAP machine was used as a measure of compliance. After a minimum period of 6 months of CPAP, all baseline examinations were repeated.

\section{Materials and methods}

\section{Polysomnography}

PSG data were recorded with a commercially available monitoring system (Mepal, MAP Medizin-Technologie, Martinsried, Germany, now, ResMed). Three channels of electroencephalography (EEG), eyes, chin and legs electromyography (EMG), thoracic and abdominal effort bands, body position, nasal pressure, nasal and oral thermistor signals and pulse oximetry were recorded together with video signals and acoustic data. Manual analyis with the Rembrandt software, which combines comprehensive high-tech PSG with flexibility for the review and analysis of sleep, was performed. In the evening preceding the sleep study, each participant was offered a standardized meal at 1800 hours. Lights were turned off at 2200 hours. Minimum recording time was $6 \mathrm{~h}$ in order to meet diagnostic standards. All sleep studies were analyzed by an experienced physician. Main outcome variables for the diagnosis of OSAS were apnea-hypopnea index (AHI) defined as the number of apneas ( $>80 \%$ reduction of the flow signal and hypopneas, $>50 \%$ reduction of the flow signal accompanied by a desaturation $>3 \%$ or by a respiratory-induced EEG arousal) per hour of sleep monitored and the oxygen desaturation index (ODI) defined as the hourly rate of episodes of arterial oxygen desaturations $>3 \%$ of a stable baseline. The severity of sleep apnea was graded as mild (5-15 events per 
hour) moderate (16-30 events per hour), and severe $(>30$ events per hour) [19].

Nasal CPAP-ventilation and assessment of compliance

Patients who were eligible for CPAP were instructed by trained nurses in the sleep center. After an AutoCPAP night (Sullivan Autoset T), a constant CPAP pressure was set at the 90-95 percentile of pressure applied by the AutoCPAP device. Clinical assessment by changes in clinical symptoms, outpatient pulse oxymetry (KonicaMinolta Pulsox 3, Anandic, Switzerland) at the patient's home, and stored data of the CPAP device were used to judge on the ongoing efficacy of the fixed CPAP pressure applied. In the case of any doubts concerning efficacy, an in-lab PSG on CPAP was performed.

In addition, one single experienced pneumonologist (ODS) reviewed all patient charts and oximetry results. The pulse oximetry data was evaluated by visual inspection for consistency of the stored overnight pulse and oxygen saturation curves. The ODI was calculated for the time period with no artifact signals recorded in the traces of the respective curves. Based on clinical follow-up information, overnight oximetry data and objective compliance information (an average of $\geq 4 \mathrm{~h}$ of CPAP use per night) was the minimum requirement to classify a patient as compliant [20]; patients were graded into two categories: nonadherent and adherent.

Clinical assessment of body composition and MRI imaging

Weight was measured to the nearest $0.1 \mathrm{~kg}$ on a calibrated scale and height was determined using a wall-mounted stadiometer. Body mass index (BMI) was calculated as the weight divided by the square of height (kilograms per square meter). Waist circumference (WC) was defined as the total circumference (centimeter) above the iliac bones. Clinical body composition data were collected from single measurements of trained nurses. Data from abdominal MRI imaging studies from a previous report [13] were used to describe the association of visceral fat areas with TNFA.

\section{Laboratory assays}

The morning after PSG and after at least 6 months of CPAP treatment between 8 and 10A.M. fasting blood (EDTA, plasma) was drawn and kept at $-80^{\circ} \mathrm{C}$ until further analyses. Serum electrolytes and glucose were assessed by an automated analyzer (Synchron, Unicel DXC 800, Beckman Coulter, Inc., Fullerton, CA 92835, USA) in collaboration with an ISO-certified clinical core laboratory. Coefficient of variation (CVs) for each analysis were $\leq 2 \%$. TNFA serum concentrations were determined with an ultrasensitive ELISA with a sensitivity $<0.17 \mathrm{ng} / \mathrm{l}$ and a range of 1.56-100 ng/l (Invitrogen Corporation, Camarillo, CA 93012, USA). This assay has an intra-assay CV of 5\% and an inter-assay $\mathrm{CV}$ of $7 \%$ respectively. Identical analyses were redone after at least 6 months of CPAP.

\section{Statistics}

Statistical analyses were done with Stata (Stata, Stata Corp. 2005. Stata Statistical Software: Release 9. College Station, TX, USA). Summary statistics are described as means and standard deviations (SD) or standard errors of the mean (SE). Baseline associations of TNFA with measures of disease severity were assessed with simple linear regression analysis after log transformation where data were skewed. Patients were first analyzed as a whole and then divided into compliant and noncompliant groups, and identical analyses were repeated within each group. Finally, we used linear regression to examine the association of ventilation and changes in TNFA levels at the end of our study. A $p$ value of less than 0.05 was considered statistically significant.

\section{Results}

Patients

Out of 566 patients screened for participation, 101 patients were excluded due to language barriers. One hundred ninety-eight patients did not give informed consent for personal and work-related reasons (difficulty to leave the job for additional study appointments). Nonconsenters and consenters did not differ by age, sex, BMI, smoking status, and socio-demographic data; however, consenters more often reported alcohol use $(215 / 260,82.7 \%$ vs. $143 / 192,74.5 \%, p=0.03)$ and had a slightly higher mean ESS score (11.3 \pm 4.4 vs. $10.6 \pm 4.8, p$ $=0.03$ ) (data not shown). Eight patients with diabetes on regular oral medication (sulfonylurea or metformin) were included. Patients were equally distributed between severity and age categories (chi-square test $p=0.4$, data not shown). Ninety five of 267 patients who agreed to participate had no indication for and, 11 patients did not start with CPAP. Twenty eight of 161 patients who started CPAP discontinued within the first month. A total of 51 patients opted to not participate until the final visit of the trial; thus, a total of 82 women and men entered the final visit. Two sets of blood samples were not available in 16 patients. We here report data on OSAS and the effects of 
CPAP on TNFA serum concentrations in 66 patients (12 women, 54 men) with a mean age of $52.3 \pm 9.8$ years treated with CPAP for at least 6 months who had no significant changes in abdominal subcutaneous fat, visceral fat, or total body fat mass after a mean of 7.8 months of CPAP therapy [13].

Baseline characteristics of ventilated study population

At baseline, women and men did not differ by age, AHI, ODI, mean Charlson score, BMI, WC, and TNFA levels. In contrast, women had significantly smaller abdominal visceral fat areas than men $\left(-50.6 \mathrm{~cm}^{2} p=\right.$ 0.03 ). Furthermore, we found no association between smoking status and baseline TNFA serum levels (ANOVA F $1.79, p=0.17$, data not shown). When grouped by adherence, compliant patients had a significantly higher baseline AHI $\left(45 \pm 26 \mathrm{~h}^{-1}\right.$ vs. $\left.28 \pm 21 \mathrm{~h}^{-1} p=0.02\right)$, and a higher ODI $\left(28 \pm 27 \mathrm{~h}^{-1}\right.$ vs. $\left.13 \pm 9 \mathrm{~h}^{-1} p=0.02\right)$, while there were no statistically significant differences regarding body composition, comorbidity, or serum TNFA concentrations (Table 1).

Baseline associations of body composition and PSG results with TNFA concentration

At baseline, we found no significant associations of $\log$ ODI or log percent time spent below an oxygen saturation of $<90 \%$ with serum TNFA concentration in any sex or group (data not shown).

Effects of CPAP on serum TNFA by adherence

Fifty compliant patients underwent effective (nightly usage $\geq 4 \mathrm{~h}$, mean $5.2 \pm 1.7 \mathrm{~h}$ ) CPAP therapy over an average period of
$7.8 \pm 1.3$ months, whereas the mean CPAP use in 16 noncompliant patients ( 3 women, 13 men) was $2.5 \pm 1.0 \mathrm{~h}$ ( $p=$ 0.00001 ) (Figs. 1 and 2). By visual inspection of the $50 \mathrm{TNF}$ pairs, we observed a TNFA increase in two individuals $(1 \mathrm{w} / 1 \mathrm{~m})$. Clinical chart review in these individuals revealed no signs for active inflammation or other reasons for such an increase at follow-up. After elimination of these two persons, the overall mean change $-0.38 \pm 0.56$, ( $p$ $=0.0001)$ in TNF was slightly higher but still significant when compared to the $t$ test performed with 50 patients $-0.28 \pm 0.71,(p=0.007)$. We thus decided to report data on all 50 patients. In this cohort, TNFA levels decreased significantly in men $(-0.49 \pm 0.13 \mathrm{ng} / \mathrm{l} ; p=0.0012)$, but not in women. When grouped by adherence, mean TNFA decreased similarly in 41 compliant $(-0.46 \pm 1.1 \mathrm{ng} / \mathrm{l} ; p=$ $0.001)$ and in 13 non-compliant $(-0.63 \pm 0.77 \mathrm{ng} / \mathrm{l} ; p=0.001)$ men and simple linear regression revealed a small but significant direct association of hours of nightly CPAP use with changes in TNFA levels $\left(R^{2} 0.08, p=0.04\right)$, whereas serum electrolytes, creatinine and fasting glucose did not change significantly at follow-up (data not shown).

\section{Discussion}

In this prospective observational study in women and men with OSAS, we found that baseline ODI and time spent below saturation $>90 \%$ during a diagnostic PSG were not predictive of serum TNFA concentration, and that an average of 8 months of CPAP decreased TNFA in men but not in women. In addition, we demonstrated that mean serum TNFA concentrations decreased in both, men with and without good adherence to CPAP use.

While a previous study reported a significant correlation of TNFA levels with ODI, ESS scores, and total cholesterol

Table 1 Baseline description of study population

\begin{tabular}{|c|c|c|c|c|c|c|c|c|}
\hline & All $(N=66)$ & SD & Women $(n=12)$ & $\mathrm{SD}$ & Men $(n=54)$ & SD & Sex difference & $p$ value \\
\hline Age (years) & 52.3 & 9.8 & 55.2 & 11.5 & 51.7 & 9.4 & 3.5 & 0.3 \\
\hline $\mathrm{AHI}^{\mathrm{a}}$ & 39.7 & 26.8 & 30.8 & 30.8 & 41.5 & 25.8 & -10.8 & 0.2 \\
\hline $\mathrm{ODI}^{\mathrm{b}}$ & 25.1 & 24.6 & 21.0 & 26.0 & 26.0 & 24.5 & 0.5 & 25.1 \\
\hline Charlson score & 0.2 & 0.5 & 0.1 & 0.2 & 0.2 & 0.5 & 0.6 & 0.2 \\
\hline BMI $\left(\mathrm{kg} / \mathrm{m}^{2}\right)^{\mathrm{c}}$ & 29.7 & 4.4 & 31.0 & 6.9 & 29.5 & 3.7 & 1.5 & 0.3 \\
\hline Waist circumferene $(\mathrm{cm})$ & 104.2 & 10.9 & 106.5 & 109.1 & 103.0 & 83.0 & 3.5 & 0.2 \\
\hline Abdominal visceral Fat $\left(\mathrm{cm}^{2}\right)$ & 193.3 & 95.6 & 153.3 & 52.0 & 202.8 & 77.4 & -50.6 & 0.03 \\
\hline TNFA (ng/l) & 1.7 & 1.0 & 1.4 & 0.7 & 1.8 & 1.1 & -0.4 & 0.2 \\
\hline
\end{tabular}

\footnotetext{
${ }^{a}$ Apnea-hypopnea index

${ }^{\mathrm{b}}$ Oxygen desaturation index

${ }^{\mathrm{c}}$ Body mass index
} 
Fig. 1 Dot plot of TNF-alpha concentrations (ng/l) at baseline (triangles) and follow-up (circles) in 50 patients adherent to CPAP (upper panel) and 16 patients without adherence (lower panel). The TNF-alpha serum concentration is represented on the $\mathrm{X}$-axis. Each horizontal line depicts a set of two blood samples of the same patient
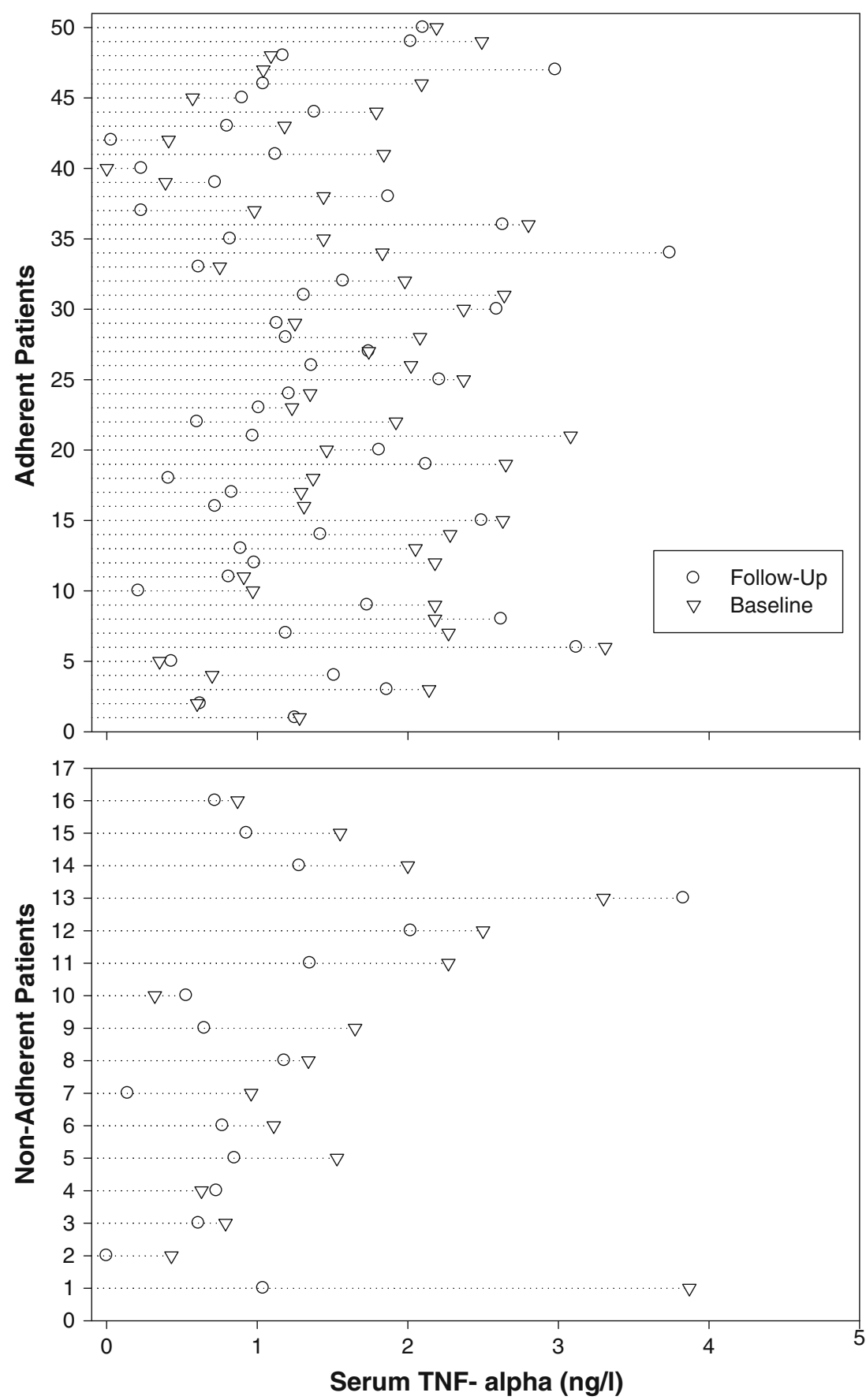

using a multiple regression model [21], we found no correlation of TNFA with ODI in our patients. Although PSG is the gold standard for the diagnosis of OSAS, data derived from a single examination may not reflect overall hypoxemia burden associated with this disease and, Ryan and colleagues [21] reported that their patients were highly selected, while our study population was recruited from an unselected clinical population. In addition, the association between inflammatory markers and disease severity is still controversial given that a mean ODI of $10 \mathrm{~h}^{-1}$ did not predict CRP and interleukin -6 levels when compared with obese controls [22].

That an average of 8 months of CPAP decreased TNFA in adherent men is in line with recent authors who have reported similar changes after 1 [9] and 2 [10] months. Effects of a comparable magnitude have been observed in a group of men after 6 weeks of CPAP use with an average duration of $4.5 \mathrm{~h} /$ night in 49 men with mild (AHI $15 \pm 8 \mathrm{~h}^{-1}$ ) to severe $\left(57 \pm 21 \mathrm{~h}^{-1}\right)$ disease [21], whereas another investigation did not find an effect of 


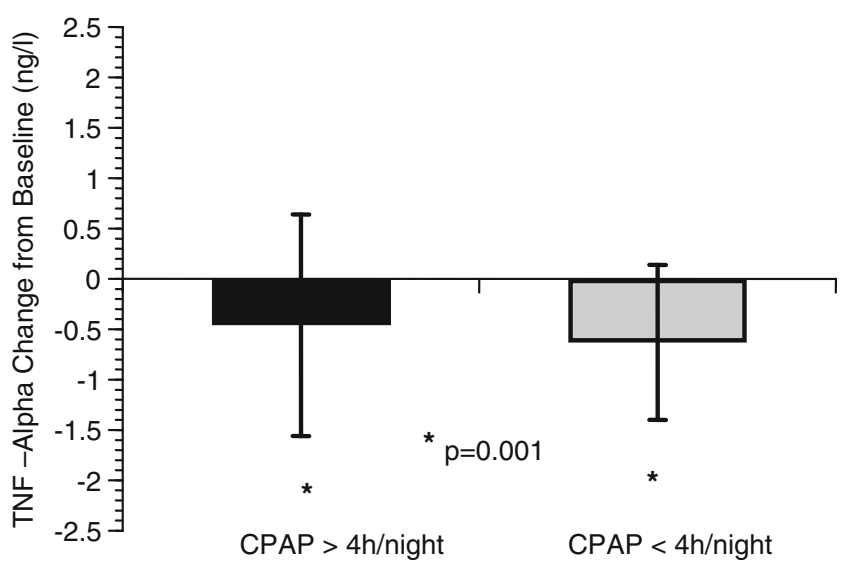

Fig. 2 Long-term effects of CPAP on mean TNFA concentrations in men grouped by CPAP use. Black Bars depict men $>4$ h of CPAP use per night, grey bars indicate men $<4 \mathrm{~h}$ of CPAP use per night. Asterisks indicate significant changes compared with baseline

CPAP on TNFA but a decrease in soluble TNFA receptor after 6 weeks of treatment [23]. Taken together, our data indicate that CPAP use of up to 8 months is beneficial to affect the inflammatory burden associated sleep apnea. Nevertheless, two patients with good adherence had an increase in TNFA serum concentrations for unclear reasons. If such patients differ from those with a decrease in TNFA remains to be determined.

In our study, an average duration of $2.5 \mathrm{~h}$ of CPAP use significantly decreased mean TNFA concentrations. Given that our non-adherent men had a lower mean AHI scores $\left(28 \pm 21 \mathrm{~h}^{-1}\right)$ compared with patients with low adherence reported from another study $\left(63 \pm 18 \mathrm{~h}^{-1}\right)$ [10], we speculate that men with fewer apneas and desaturations per night might well benefit from a shorter nightly CPAP use.

While a growing body of evidence supports a link between OSAS and systemic inflammation [4, 24], the underlying mechanisms are not completely understood. In vitro studies have suggested that sustained hypoxia leads to an activation of genes encoding proteins that increase delivery. On the other hand, hypoxemia is associated with the activation of transcription factors that trigger the expression of genes that encode inflammatory proteins such as TNFA [25]. Reversal of hypoxemia by CPAP thus leads to a downregulation of such transcription factors.

Although visceral obesity may also be a contributor to inflammation in OSAS [26], data on the effects of CPAP on visceral adipose tissue and inflammatory cytokines are sparse. Authors have either published a reduction in visceral fat mass after CPAP without data on inflammatory cytokines [12] or changes in cytokines without addressing visceral fat $[9,23,27]$.

While we reported earlier that visceral fat mass was unaffected by CPAP [13], we now demonstrate a decrease in TNFA after CPAP use. Both results confirm an earlier report [24]. Our findings that CPAP decreased TNF independent of changes in adipose tissue suggest that intermittent hypoxia [21, 27], the immediate decrease in sympathetic output [28] have stronger impact upon inflammatory cytokines than fat. A decrease in TNFA after 6 months septoplasty and uvulo-palato-pharyngoplasty without changes in BMI supports this hypothesis [29].

Our observation that TNFA decreased even in patients using CPAP for less than $4 \mathrm{~h} /$ night and who had a lower AHI, and thus, a less severe disease suggests that the effect of CPAP on TNFA could also be a function of disease severity. Given that low adherence is frequently associated with low severity [20] and that we found a small but significant dose-response relationship of nightly CPAP use with changes in TNFA levels, our data suggest that also men with low AHI at baseline benefit from CPAP use.

Our study has several limitations. First, we did not include a control group and the effects of CPAP. However, given the beneficial effects of CPAP on a large variety of cardiovascular outcomes variables [30] and in addition on quality of life [31] during the initial design of the study and from a clinical perspective, it seemed unethical to withhold ventilation to a larger patient population over such a long period of time: To date, several sham trials with a duration of up to 6 months are underway. In addition, the number of women studied and the number of non-compliant patients is relatively small and results in non-compliant persons are likely to differ from patients in a true control or sham ventilation group. Therefore, conclusions drawn for women should be interpreted with caution, and the small sample size might have influenced our results. Furthermore, this observational study was initially planned to assess changes in body composition after 6 months of CPAP use. Thus, our sample size and the different mean baseline ODI and AHI data between the two groups might have been underpowered to detect the effect of adherence with CPAP on TNFA. However, studies on the effects of CPAP on visceral fat or TNF, either report results in men or did not report the sex of the patients $[12,24,32]$, while others included a very small number of women [10] $(n=3)$.

In summary, our study demonstrated that baseline hypoxemia during PSG did not predict serum TNFA concentrations, but 8 months of CPAP decreased serum TNFA without - as previously shown, affecting body fat mass suggesting that other factors than adipose tissue or reversal of hypoxia modulate the beneficial effects of CPAP on inflammatory cytokines. Future studies should attempt to recruit more women and additionally assess longitudinal changes in body composition, inflammation, and metabolism in both genders affected by this disease. Given that CPAP decreased TNFA also in men with low adherence and the fact that we found a small but significant association of nightly 
CPAP use with changes of TNFA, patients with lower adherence should be motivated to use CPAP as frequently and long as possible.

Acknowledgments The authors thank Sandro Pampallona, Formed (Statistics for Medicine) Evolène for his important statistical comments on the manuscript, his engagement in the study design, power calculation, and the design of the database. We also appreciate the technical expertise and the assistance of the staff members of the Institute of Radiology for performance of the MRI scans and Clinical Chemistry of the Kantonsspital St. Gallen for the performance of the assays. The support by the staff of the Kantonsspital St. Gallen Multidisciplinary Center for Sleep Medicine for the support with patient recruitment and collection blood samples as well as Drs. Ullmer, Knoblauch, Nierhoff and Paky are appreciated. Dr. Korte was supported by the Institute for Clinical Chemistry and Hematology and an ALTANA research price grant. The TNF concentration measurements and polymorphism determinations represent a large part of Mrs. Hahn's diploma thesis. Dr. Münzer was supported by a Forschungskolleg Geriatrie Grant of the Robert Bosch Foundation, Stuttgart, Germany.

Conflict of interest The authors declare that they have no conflicts of interest.

\section{References}

1. Krueger JM (2008) The role of cytokines in sleep regulation. Curr Pharmaceut Des 14(32):3408-3416

2. Bresnitz EA, Goldberg R, Kosinski RM (1994) Epidemiology of obstructive sleep apnea. Epidemiol Rev 16(2):210-227

3. Entzian P, Linnemann K, Schlaak M, Zabel P (1996) Obstructive sleep apnea syndrome and circadian rhythms of hormones and cytokines [see comments]. Am J Respir Crit Care Med 153 (3):1080-1086

4. Punjabi NM, Beamer BA (2007) C-reactive protein is associated with sleep disordered breathing independent of adiposity. Sleep 30 (1):29-34

5. Ciftci TU, Kokturk O, Bukan N, Bilgihan A (2004) The relationship between serum cytokine levels with obesity and obstructive sleep apnea syndrome. Cytokine 28(2):87-91

6. Vgontzas AN, Papanicolaou DA, Bixler EO, Kales A, Tyson K, Chrousos GP (1997) Elevation of plasma cytokines in disorders of excessive daytime sleepiness: role of sleep disturbance and obesity. J Clin Endocrinol Metab 82(5):1313-1316

7. Vgontzas AN, Papanicolaou DA, Bixler EO, Hopper K, Lotsikas A, Lin HM, Kales A, Chrousos GP (2000) Sleep apnea and daytime sleepiness and fatigue: relation to visceral obesity, insulin resistance, and hypercytokinemia. J Clin Endocrinol Metab 85 (3):1151-1158

8. Vgontzas AN, Zoumakis E, Lin HM, Bixler EO, Trakada G, Chrousos GP (2004) Marked decrease in sleepiness in patients with sleep apnea by etanercept, a tumor necrosis factor-alpha antagonist. J Clin Endocrinol Metab 89(9):4409-4413

9. Minoguchi K, Tazaki T, Yokoe T, Minoguchi H, Watanabe Y, Yamamoto M, Adachi M (2004) Elevated production of tumor necrosis factor-alpha by monocytes in patients with obstructive sleep apnea syndrome. Chest 126(5):1473-1479

10. Dorkova Z, Petrasova D, Molcanyiova A, Popovnakova M, Tkacova R (2008) Effects of continuous positive airway pressure on cardiovascular risk profile in patients with severe obstructive sleep apnea and metabolic syndrome. Chest 134(4):686-692
11. Kobayashi K, Nishimura Y, Shimada T, Yoshimura S, Funada Y, Satouchi M, Yokoyama M (2006) Effect of continuous positive airway pressure on soluble cd40 ligand in patients with obstructive sleep apnea syndrome. Chest 129(3):632-637

12. Chin K, Shimizu K, Nakamura T, Narai N, Masuzaki H, Ogawa Y, Mishima M, Nakao K, Ohi M (1999) Changes in intra-abdominal visceral fat and serum leptin levels in patients with obstructive sleep apnea syndrome following nasal continuous positive airway pressure therapy. Circulation 100(7):706-712

13. Münzer T, Hegglin A, Stannek T, Schoch OD, Korte W, Buche D, Schmid C, Hurny C (2010) Effects of long-term continuous positive airway pressure on body composition and igfl. Eur $\mathrm{J}$ Endocrinol 162(4):695-704

14. Vgontzas AN (2008) Does obesity play a major role in the pathogenesis of sleep apnoea and its associated manifestations via inflammation, visceral adiposity, and insulin resistance? Arch Physiol Biochem 114(4):211-223

15. Schiza SE, Mermigkis C, Panagiotis P, Bouloukaki I, Kallergis E, Tzanakis N, Tzortzaki E, Vlachaki E, Siafakas NM (2010) Creactive protein evolution in obstructive sleep apnoea patients under CPAP therapy. Eur J Clin Investig 40(11):968-975

16. Bloch KE, Schoch OD, Zhang JN, Russi EW (1999) German version of the Epworth Sleepiness Scale. Respiration 66(5):440-447

17. Douglass AB, Bornstein R, Nino-Murcia G, Keenan S, Miles L, Zarcone VP Jr, Guilleminault C, Dement WC (1994) The sleep disorders questionnaire. I: creation and multivariate structure of sdq. Sleep 17(2):160-167

18. Charlson ME, Pompei P, Ales KL, MacKenzie CR (1987) A new method of classifying prognostic comorbidity in longitudinal studies: development and validation. J Chron Dis 40(5):373-383

19. Flemons WW, Buysse D, Redline S, Pack A, Strohl K, Wheatley J, Young T, Douglas N, Levy PWM, Fleetham J, White D, Schmidt-Nowarra W, Carley D, Romaniuk J (1999) Sleep-related breathing disorders in adults: recommendations for syndrome definition and measurement techniques in clinical research. The report of an american academy of sleep medicine task force. Sleep 22(5):667-689

20. Weaver TE, Grunstein RR (2008) Adherence to continuous positive airway pressure therapy: the challenge to effective treatment. Proc Am Thorac Soc 5(2):173-178

21. Ryan S, Taylor CT, McNicholas WT (2006) Predictors of elevated nuclear factor-kappab-dependent genes in obstructive sleep apnea syndrome. Am J Respir Crit Care Med 174(7):824-830

22. Yokoe T, Minoguchi K, Matsuo H, Oda N, Minoguchi H, Yoshino G, Hirano T, Adachi M (2003) Elevated levels of C-reactive protein and interleukin- 6 in patients with obstructive sleep apnea syndrome are decreased by nasal continuous positive airway pressure. Circulation 107(8):1129-1134

23. Arias MA, Garcia-Rio F, Alonso-Fernandez A, Hernanz A, Hidalgo R, Martinez-Mateo V, Bartolome S, Rodriguez-Padial L (2008) Cpap decreases plasma levels of soluble tumour necrosis factor-alpha receptor 1 in obstructive sleep apnoea. Eur Respir J 32(4):1009-1015

24. Vgontzas AN, Zoumakis E, Bixler EO, Lin HM, Collins B, Basta M, Pejovic S, Chrousos GP (2008) Selective effects of cpap on sleep apnoea-associated manifestations. Eur J Clin Investig 38 (8):585-595

25. Ryan S, McNicholas WT (2008) Intermittent hypoxia and activation of inflammatory molecular pathways in osas. Arch Physiol Biochem 114(4):261-266

26. Vgontzas AN, Bixler EO, Chrousos GP (2005) Sleep apnea is a manifestation of the metabolic syndrome. Sleep Med Rev 9 (3):211-224

27. Ryan S, Taylor CT, McNicholas WT (2005) Selective activation of inflammatory pathways by intermittent hypoxia in obstructive sleep apnea syndrome. Circulation 112(17):2660-2667 
28. Phillips CL, Yang Q, Williams A, Roth M, Yee BJ, Hedner JA, Berend N, Grunstein RR (2007) The effect of short-term withdrawal from continuous positive airway pressure therapy on sympathetic activity and markers of vascular inflammation in subjects with obstructive sleep apnoea. J Sleep Res 16 (2):217-225

29. Constantinidis J, Ereliadis S, Angouridakis N, Konstantinidis I, Vital V, Angouridaki C (2008) Cytokine changes after surgical treatment of obstructive sleep apnoea syndrome. Eur Arch Otorhinolaryngol 265(10):1275-1279

30. Marin JM, Carrizo SJ, Vicente E, Agusti AG (2005) Long-term cardiovascular outcomes in men with obstructive sleep apnoea- hypopnoea with or without treatment with continuous positive airway pressure: an observational study. Lancet 365(9464):10461053

31. Pichel F, Zamarron C, Magan F, del Campo F, Alvarez-Sala R, Suarez JR (2004) Health-related quality of life in patients with obstructive sleep apnea: effects of long-term positive airway pressure treatment. Respir Med 98(10):968-976

32. Steiropoulos P, Kotsianidis I, Nena E, Tsara V, Gounari E, Hatzizisi O, Kyriazis G, Christaki P, Froudarakis M, Bouros D (2009) Long-term effect of continuous positive airway pressure therapy on inflammation markers of patients with obstructive sleep apnea syndrome. Sleep 32(4):537-543 